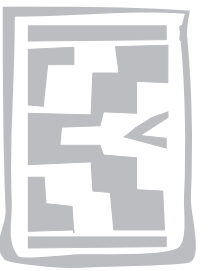

\title{
Endemic stability for Theileria parva infections in Ankole calves of the Ankole ranching scheme, Uganda
}

\author{
F.M. KIVARIA ${ }^{1 *}$, C. HEUER ${ }^{2}$, F. JONGEJAN² ${ }^{2}$ J. OKELLO-ONEN ${ }^{3}$, T. RUTAGWENDA ${ }^{4}$, \\ F. UNGER ${ }^{5}$ and W. BOEHLE ${ }^{5}$
}

\begin{abstract}
KIVARIA, F.M., HEUER, C., JONGEJAN, F., OKELLO-ONEN, J., RUTAGWENDA, T., UNGER, F. \& BOEHLE, W. 2004. Endemic stability for Theileria parva infections in Ankole calves of the Ankole ranching scheme, Uganda. Onderstepoort Journal of Veterinary Research, 71:189-195

A population-based study was carried out on the Ankole ranching scheme in south-west Uganda with the aim of determining the endemic status of Theileria parva infections. For this purpose, the age-related sero-prevalence of $T$. parva and the specific calf mortality associated with the parasite were assessed. Blood samples were collected from 931 Ankole calves of up to 12 months of age from 81 randomly selected herds. The relationship between rainfall pattern and whole-body Rhipicephalus appendiculatus counts was determined.

The influence of tick control practices on East Coast fever-related calf mortality, and sero-positivity were also determined. A significant $\left(\mathrm{r}^{2}=0.76, P=0.000\right)$ association between $R$. appendiculatus counts and rainfall was observed. There was no significant $(P>0.05)$ association between theileriosis-related calf mortality, sero-positivity and the different tick control practices. Antibody prevalence based on the PIM ELISA was above $70 \%$ among calves of 6 months of age in $96 \%$ in all the herds.

Theileria parva-related calf mortality determined by repeated herd visits and farm records ranged between $0 \%$ and $5.4 \%$. It was concluded that endemic stability for theileriosis, caused by T. parva, existed in the study area, and that the risk of the occurrence of economically important outbreaks of East Coast fever in indigenous cattle was regarded as minimal under the prevailing conditions.

Keywords: East Coast fever, endemic stability, Theileria parva, Uganda
\end{abstract}

\section{INTRODUCTION}

East Coast fever (ECF), a tick-borne protozoan disease of cattle caused by Theileria parva, occurs in

\footnotetext{
* Author to whom correspondence is to be directed

1 Population Studies, Animal Disease Research Institute, P.O. Box 9254, Dar es Salaam, Tanzania E-mail: fredkiv@yahoo.com

2 Department of Farm Animal Health, Faculty of Veterinary Medicine, Utrecht University, Yalelaan 7, 3584 CL Utrecht, The Netherlands

3 Livestock Health Research Institute, Tororo, Uganda

4 Veterinary Faculty, Makerere University, Kampala, Uganda

5 GTZ-Integrated Pastoral Development Project, Sanga, Mbarara, Uganda

Accepted for publication 27 January 2004-Editor
}

eastern and central Africa and is transmitted by the three-host ixodid tick Rhipicephalus appendiculatus. The disease is frequently fatal in susceptible Bos taurus and indigenous Bos indicus cattle outside endemic areas. The economic losses caused by ECF in the region have recently been estimated at US\$ 168 million each year (Mukhebi, Perry \& Kruska 1992). As with most tick-borne diseases (TBD) young (up to 4 months of age) calves are protected against ECF by colostrums derived maternal immunity. During this period calves are susceptible to infection, but, if infected, the ensuing disease is less severe than in older fully susceptible cattle. If infection rates are high enough to infect the majority of calves during the period of protection there will be little or no clinical theileriosis; this state of 
near equilibrium between parasite, vector and host is known as "endemic stability" (Norval, Perry \& Young 1992).

Norval et al. (1992) describe endemic stability as a "climax relationship between host, vector and environment in which all coexist with the virtual absence of clinical theileriosis, while endemic instability means an incomplete relationship in which clinical disease occurs". In the case of T. parva infections, endemic stability is defined as "the state in a cattle population in which the majority of that population becomes immune by six months of age and little or no disease occurs". This state exists when (1) the large majority of young calves ( $\leq 6$ months) are exposed to a low but continuous T. parva challenge; (2) the cattle population possesses a low innate susceptibility to the effects of T. parva infection; and (3) there is little seasonal variation in calving providing a steady presentation of a susceptible calf population to $R$. appendiculatus throughout the year. A typical ECF-endemic state is characterized by rare clinical disease with low mortality rates that are limited to young stock. This state is found in few localised parts of Africa, chiefly on the East African highlands where the local Zebu cattle are maintained under extensive management conditions with little or no efficacious acaricide application and where $R$. appendiculatus can undergo at least two generations annually (Perry 1994). Under such circumstances greater numbers of cattle are reservoirs of T. parva at low levels of parasitaemia. Consequently, ticks bear low infection rates. Calves born in these areas become immune through natural infection before they are three months old (Moll, Lohding \& Young 1984; Moll, Lohding, Young \& Leitch 1986) and therefore little or no clinical disease occurs. Parameters used to characterize endemic stability are the antibody prevalence of calves (Deem, Perry, Katende, McDermott, Mahan, Maloo, Morzaria, Musoke \& Rowlands 1993) and age specific morbidity and mortality rates (Norval et al. 1992).

Settlers in the Ankole ranching scheme (ARS) rely mainly on acaricides to control ticks. However, acaricides are rarely used correctly and tick control is inefficient. East Coast fever is the most frequent TBD in the ARS with an estimated sero-prevalence of $72 \%$ (Heuer 1995). Because calves are exposed at a young age and the vector, $R$. appendiculatus, is assumed to be present throughout the year, it is probable that endemic stability against $T$. parva exists in cattle in the ARS. If so, it might be advisable to reduce the level of tick control. This would alleviate a substantial financial burden on house- holds (Brandl, Heuer, Kiryapawo, Mugisha, Schulz \& Schwartz 1995). This study addressed the question as to whether or not endemic stability to ECF exists in the ARS cattle.

\section{MATERIALS AND METHODS}

\section{Study area and husbandry practices}

The ARS is a confined area of approximately 1500 $\mathrm{km}^{2}$ in Nyabushozi County close to Lake Mburo and Mburo National Park in Mbarara district, south-west Uganda. It is an ECF-endemic area with a bimodal rainfall pattern, with rain in March to May and September to November. The annual rainfall ranges between 750 and $875 \mathrm{~mm}$. Cattle of the Ankole breed (Bos indicus) predominate. The cattle are maintained in a semi-pastoral, open grazing system. They are housed at night in bomas constructed from thorny tree branches to protect them from predators and thieves. During the night, unweaned calves are kept inside calf sheds, thereby limiting exposure to ticks. Milking is generally done once per day in the morning until the calves are about 3 months old. After the milking, the calves are allowed to suckle, and the cattle are then taken to their grazing plots that may be several kilometres away. Young calves ( $<3$ months old) are kept near the house during the day until the herd returns in the evening. They begin to accompany the herd at approximately 3 months of age. Three types of acaricides are used in the study area, viz. Bayticol $@$ (flumethrin), Decatix $®$ (deltamethrin) and Taktic $®$ (amitraz).

\section{Sampling technique}

The reference population was all cattle in herds of the ARS. Two lists ( $A$ and $B$ ) each of 80 randomly selected herds were obtained from a sampling frame provided by the Ranch Restructuring Board. List A was the primary list for selection, list $B$ was used to replace refusals or any lost sample during the study period. All calves in a selected herd that were born up to 1 month prior to the initial farm visit were included in the study and followed up to the age of 1 year. Calves that had died within 1 month of age and before the visit of the investigation team were assumed to have died from reasons other than ECF, because most calves were assumed to have protective maternally derived immunity against ECF during that period.

The following assumptions were made for the calculation of the sample size (Heuer 1995): calving 
rate $(0.40)$; gross calf mortality rate up to 1 year $(P$ $=0.25)$; calf mortality due to ECF (0.05); no calving seasonality; average herd size (60 cows) and calves per cow per year (0.40). The sample size needed to estimate mortality due to ECF with an acceptable error $(L)$ of \pm 0.04 and 0.95 confidence was determined by the formula $\mathrm{N}=4 \mathrm{PQ} / \mathrm{L}^{2}$ (Martin, Meek \& Willeberg 1987), resulting in $N \sim 470$. Since calves belonged to different herds and mortality due to ECF was thought to vary from herd to herd, mortality was assumed to correlate within the herd. To compensate for cluster effects the sample size was therefore increased two-fold, hence the required sample size was estimated as $\mathrm{N}=940$ calves.

The herds were visited every 8 weeks. Therefore, ten herd visits per week and two herds per day on a 5-day-week basis were performed. Because the calving rate was subsequently considered to be overestimated, and in order to include all seasons, the study period was extended to 1 year. All calves born during the study period were ear-tagged at the earliest visit after birth. Farmers were requested to keep records of date, animal identity and events such as birth, deaths, treatments, sale and purchase, acaricide use and occurrence of other diseases. Missing information in the farmers' information was supplemented by the completion of questionnaires. Tick infestation was estimated by counting the number of adult $R$. appendiculatus on the whole body of each calf. Blood was collected from each calf in $10 \mathrm{~m} \ell$ sterile Vacutainer $₫$ tubes (Sherwood, UK). After collection, the blood samples were stored in an icebox until transferred to a refrigerator (usually within $4 \mathrm{~h}$ ). The following day, the sera were separated by centrifugation at $3000 \mathrm{~g}$ for 20 $\min$ and stored at $-20^{\circ} \mathrm{C}$.

All serology was carried out at the International Livestock Research Institute (ILRI), Nairobi, Kenya. Because the field investigators could not observe tick control practices (TCP) during their application, it was necessary to rely on the information supplied by the herd owners. These practices were subsequently classified into four categories, i.e. 1= "pouron" (flumethrin or deltamethrin as a pour-on formulation), as "Amitraz" (usually applied as spray, rarely as dip), the latter, based on the dilution ratios, subdivided into 2 = "correct strength" and 3 = "under strength", or as 4 = "change" (herds changing these two compounds during the study). The study period was divided into four seasons, i.e. 1= long rains (August to December 1995), 2 = short rains (April to May 1996), 3 = short dry, (June to July 1996) and 4 = long dry (January to March 1997).

\section{Serological test}

The polymorphic immunodominant molecule (PIM) recombinant antigen ELISA was used to detect antibodies to T. parva (Katende, Toye, Skilton, Nene, Morzaria \& Musoke 1997). The ELISA results were expressed as percent positive (PP) values (Wright, Nilsson, Van Rooij, Lelenta \& Jeggo 1993). The ELISA has been estimated to have a sensitivity of $99 \%$ and a specificity of $97 \%$ (Katende et al. 1997). Any reading of $15 \mathrm{PP}$ or higher was considered positive.

\section{Statistical analysis}

Continuous PP values were regressed on age and TCP as main effects and season of birth and herdsize as potential confounders in a generalized linear model (SPSS for Windows, release 9.0.0). The farm was entered into the model as a random effect to account for herd clustering (McDermott, Schukken \& Shoukri 1994). A preliminary check of the data revealed age polynomials of up to the $4^{\text {th }}$ order to be more appropriate than the assumption of a straight-line association between age and PP. The age polynomials were therefore added to the model. Variables were considered significant at $P \leq 0.05$. Predicted values from the generalized linear model were plotted versus age to show the trend of PP relative to age up to 1 year.

Calf mortality was classified as caused by ECF according to farmer observations, or as caused by some other reason. East Coast fever's cumulative mortality (CM) was defined as the number of calves dying during an age-month divided by the number of calves starting the month minus half withdrawals unrelated to the cause of death (Thrusfield 1995). Mean CM and $95 \%$ confidence intervals were calculated for each month of age. To compare ECFrelated calf mortality between month of age, between season of birth and between the four TCP strata, an overall chi-square and pair-wise risk-rate differences (RRD) were calculated (Rothman \& Greenland 1998). Survival table analysis (Kramer 1988) was used to compare survival curves for ECF-related mortality with mortality due to other reasons. The survival curves were compared using the log rank test. Calves that were slaughtered, consumed, given away or lost to follow-up were treated as censored observations. The Pearson product moment correlation coefficient $\left(r^{2}\right)$ was used to quantify the relationship between the rainfall pattern and the whole-body $R$. appendiculatus counts. 


\section{RESULTS}

\section{Descriptive results and distribution of risk factors}

From 931 calves that entered the observation period, 122 calves were censored during the follow-up period of 12 months (sold, slaughtered, donated, or lost). Among these, 738 had one or more ELISA results yielding a total of 1378 serum titres. Samples from 71 calves were discarded. The 738 calves were believed to be a random subset of the total calves studied. Table 1 summarizes the distribution of both herds and calves within the type of acaricide commonly used in the study area. A total of 81 herds, containing between five and 26 calves were recruited for the study. No farmer declined to participate in the study. Between January and April 1996 the study was interrupted for logistical and organisational reasons. However, the disruption had no major effect on the study. Calving seasonality was not observed. The observed whole-body $R$. appendiculatus counts were significantly associated $\left(\mathrm{r}^{2}=0.76, P=0.000\right)$ with the recorded rainfall.

\section{ECF-related mortality}

Up to the age of 12 months, 31 calves died from ECF, while 29 died for other reasons. Adjusted for time at risk, the overall cumulative calf mortality of ECF and that for other reasons until 1 year of age were $0.054 \pm 0.01$ and $0.053 \pm 0.01$, respectively. Thus, ECF contributed $50 \%$ to the overall calf mortality. The ECF and non-ECF mortality rates were not statistically different (log rank- $\chi^{2}=0.016,1 \mathrm{df}$ ). The ECF mortality was restricted to $21 \%$ of all herds in which it varied from $4 \%$ to $67 \%$, while $79 \%$ of the herds had no ECF deaths. The intra-cluster correlation and variance inflation factor for calf mortality were 0.09 and 1.74, respectively. Applied to this resulted in an observed precision level of 0.023 , as compared to the a priori required minimum precision of 0.04 . The increase in precision was due to the extended observation period. The highest proportion of calves that died from ECF was 3 months of age. Fig. 1 shows the cumulative calf mortality of ECF. There was a significant association between age and ECF mortality $(\chi 2=89.6,3 \mathrm{df}, P<0.001)$; $29 \%$ of ECF deaths occurred during the first 3 months of life and $71 \%$ of deaths occurred up to 6 months of age. The association between ECF specific calf mortality and season of birth was significant $\left(\chi^{2}=39.9,3 \mathrm{df}, P<0.0001\right)$. The observed cumulative ECF mortality ( \pm standard error) in each season was $0.06 \pm 0.01,0.05 \pm 0.02,0.02 \pm 0.01$ and $0.004 \pm 0.004$ for the long rain, short rain, short dry and long dry seasons, respectively. Calf mortality due to ECF did not differ significantly between the TCP strata. The ECF cumulative mortality was $0.02 \pm 0.01,0.04 \pm 0.01$ and $0.07 \pm 0.02$ for the "correct-strength", "under-strength" and "change" categories, respectively. No calf deaths from ECF were observed in the "pour-on" category. The results from RRD are shown in Table 2.

\section{Theileria parva antibody prevalence}

Average T. parva antibody responses in the Ankole calves between October 1995 and April 1997 are shown in Fig. 2. The overall ECF challenge was high as $547(74 \%)$ of the calves were positive at the age $\geq 6$ months. The mean $T$. parva antibody titres were $46.4 \pm 8.4,37.9 \pm 9.0,33.0 \pm 9.0$ and $41.9 \pm$ 9.0 for the four seasons, respectively. The seasonal means were significantly different $(P<0.001)$. There were no significant pair-wise differences between the mean PP by TCP. The mean PP with their respective standard error were $47.75 \pm 33.55$, $37.50 \pm 17.5,34.70 \pm 7.0$ and $39.81 \pm 14.2$ for the "pour-on", "correct-strength", "under-strength" and "change" strata, respectively. Mean T. parva antibody titres adjusted for age, TCP, season of birth and herd size were significantly different among the 81 herds $(P<0.001)$. The proportion of calves that were positive to $T$. parva ELISA (i.e. $\geq 15$ PP) by TCP was $72 \%$ (pour-on), $85 \%$ (correct strength), $80 \%$ (under-strength) and $85 \%$ (change). The GLM results indicated that calf age, season of birth and herd were significantly associated with variations in antibody prevalence to T. parva. Age and three polynomials of age fitted the data best. The trend line of Fig. 2 shows that PP was high at birth, dropped to the lowest level at 4 months of age, then increased sharply until 9 months and remained constant thereafter.

\section{DISCUSSION}

Endemic stability for ECF is more likely to exist where the prevalence of serum antibodies to $T$. par$v a$ is high $(\geq 70 \%)$, while an unstable state is characterized by low $(<30 \%)$ antibody prevalence (Norval et al. 1992; Perry \& Young 1995). Moreover, a stable state is characterized by at least $40 \%$ seroprevalence among calves up to 6 months of age (FAO 1986). Likewise, endemic stability is characterized by mortality between $0-1.4 \%$, confined mainly to calves. Mortality incidences in the order of $5-10 \%$ and $20 \%$ or above are characteristic fea- 
F.M. KIVARIA et al.

TABLE 1 Farms and calf distribution in the Ankole ranching scheme with respect to tick control practices and the type of acaricide used

\begin{tabular}{|l|l|l|l|l|}
\hline \multicolumn{2}{|l|}{ Application category } & \multicolumn{2}{l|}{} \\
\hline \multirow{2}{*}{ Acaricide type } & \multicolumn{2}{l|}{ Under strength } & Right strength & Calves \\
\cline { 2 - 5 } & Farms & Calves & Farms & 63 \\
\hline Bayticol \& Decatix" & - & - & 8 & 303 \\
Amitraz & 45 & 478 & 19 & - \\
"Change" & 9 & 87 & - & 366 \\
\hline Total & 54 & 565 & 27 & 3 \\
\hline
\end{tabular}

1 Used as pour-on preparations

2 Usually applied as a spray wash, rarely a dip

TABLE 2 East Coast fever-related calf mortality risk-rate differences (RRD), pair-wise comparison for the different tick control practices strata

\begin{tabular}{|l|l|l|l|l|}
\hline Comparison & RRD & SE $^{\#}$ (RRD) & $\chi$ score & $P$ value \\
\hline Right strength $^{\text {a/pour-on }}{ }^{\mathrm{b}}$ & 0.026 & 0.013 & 1.025 & 0.153 \\
Under strength/pour-on $^{\text {Change } / \text { pour-on }}$ & 0.045 & 0.011 & 1.354 & 0.089 \\
Under strength/right strength & 0.095 & 0.029 & 1.967 & 0.246 \\
Change/right strength & 0.019 & 0.017 & 1.017 & 0.155 \\
Change/under strength & 0.069 & 0.031 & 2.397 & 0.083 \\
\hline
\end{tabular}

\# Standard error of the mean

a Farmers who use the correct strength of the acaricide

b Farmers who use Bayticol and/or Decatix

c Farmers who over-dilute the acaricide

d Farmers who switch between the pour-on preparations and Amitraz

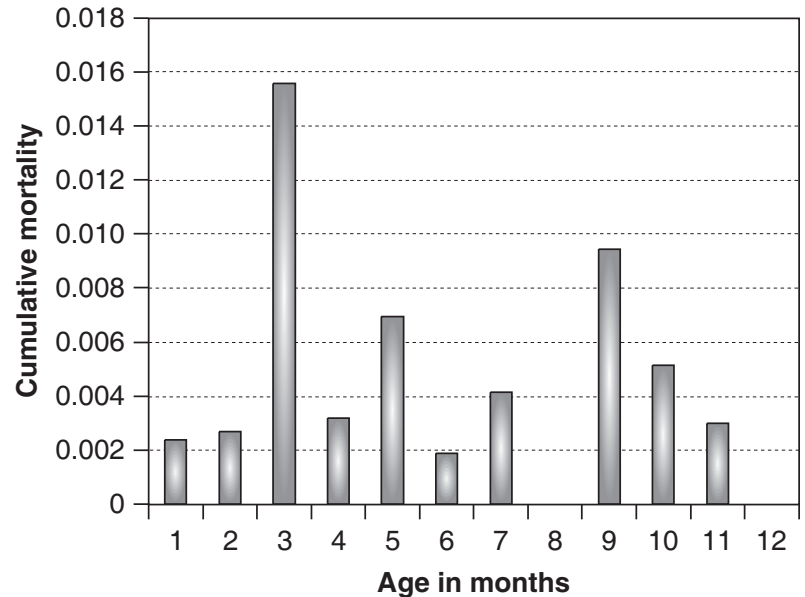

FIG. 1 East Coast Fever cumulative calf mortality by month of age in the Ankole ranching scheme between October 1995 and April 1997

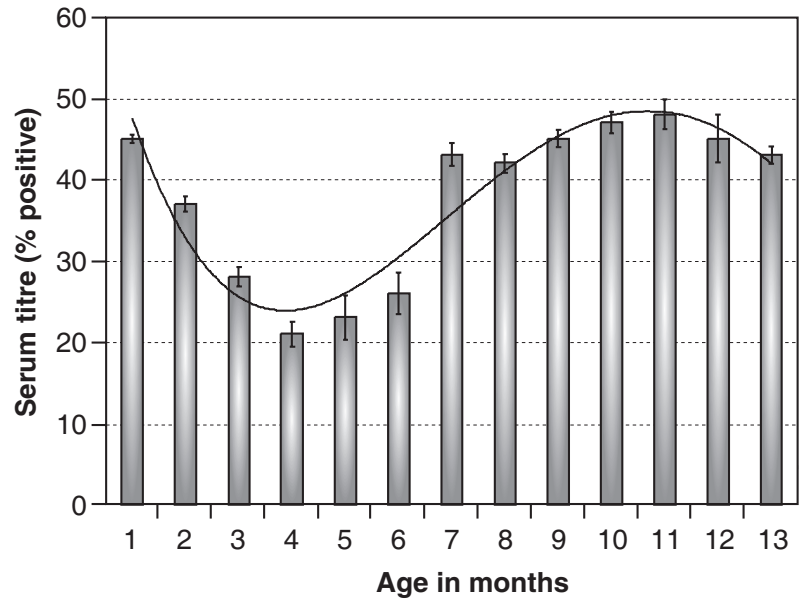

FIG. 2 Mean T. parva antibody titres (percent positive values with standard error of the mean) of the Ankole calves in the Ankole ranching scheme between October 1995 and April 1997. The line is a polynomial trend line for mean predicted percent positive values 
tures for endemically unstable and epidemic states (FAO 1986). According to these definitions $96 \%$ (78/81) of all herds investigated in this study were considered to be in a state of endemic stability as the serum antibody prevalence was over $70 \%$ from 6 months of age onwards. This implies that the pool of susceptible animals may be too small for an outbreak of ECF.

The herd effect on sero-prevalence may be explained by differences in management such as communal grazing, distance to and tick infestation at watering points, herd locations, differences in the age and night housing of calves. Serum antibody prevalence was highly correlated with age and to a lesser extent with season of calving. High titres at birth which decline until 3 months of age reflect a typical pattern of a decay of initially high maternally-derived antibodies. After weaning at about 3-4 months of age, the serum titres increased, probably because of increased exposure to infected ticks after the calves had started to accompany their dams for grazing. As antibodies were lowest at 3-4 months of age, ECF specific mortality was highest and tended to decline as antibodies increased sharply.

The overall incidence of mortality due to ECF up to 12 months of age (5.4\%) was higher than expected in an endemically stable state (Norval et al. 1992) even though ECF mortality only affected $29 \%$ of the study herds. A possible explanation for this high mortality is a bias in the diagnosis. In this study, incentives were offered to farmers for bringing calves suspected to have died from ECF to the local veterinary office. However, this strategy was not successful. Thus, we had to rely on the herders for information on the suspected cause of mortality. East Coast fever was the most popular of all calf diseases due to a long history of acaricide use in Uganda and herders are obliged by law to use acaricides against tick infestations. Because of the high awareness of the herders for ECF, a number of calf deaths for other reasons were presumed to be misclassified as ECF. Our overall estimate of ECFrelated mortality was therefore probably too high. However, the bias was unlikely to affect the agerelated pattern. Consequently, the apparent high mortality was not considered as reliable evidence against endemic instability. Nevertheless, we think that some true ECF-related calf mortality did occur and was possibly facilitated by starvation stress, parasite infestation and other diseases, for example Escherichia coli infection (Moll et al. 1984, 1986).
The TCP categories were very crude and probably did not adequately reflect the differences in tick control. However, the conditions of this study did not allow a more refined description. This was probably one reason for the lack of statistically significant differences in ECF-related mortality as well as seropositivity between the TCP strata. In addition, because tick control is prescribed by law, information from farmers on the effectiveness of tick control in their herds may have been exaggerated. The study team had the unanimous view that tick control was largely inefficient throughout the ARS.

Lack of calving seasonality, permanent $R$. appendiculatus challenge and high sero-prevalence to $T$. parva antibody at an early age strongly suggested endemic stability in the cattle population of the ARS. Consequently, cattle herders in the scheme could save the very considerable cost of tick control (Heuer 1995).

\section{ACKNOWLEDGEMENTS}

The study was designed and implemented by a project of Uganda-German bilateral co-operation. The authors acknowledge the financial and managerial contributions of the Ministry of Agriculture and Fisheries, Government of Uganda, and the Deutsche Gesellschaft für Technische Zusammenarbeit (German Agency for Technical Co-operation). Our colleagues are thanked for critically reading the manuscript and for their very useful comments. We are particularly grateful to Drr R.J. Dalgliesh and J. Molloy of Queensland Department of Primary Industries, Australia, for their constructive criticism and helpful suggestions. We also extend special thanks to the farming community of ARS for their willingness to co-operate and participate in the study.

\section{REFERENCES}

BRANDL, F.E., HEUER, C., KIRYAPAWO, T., MUGISHA, A., SCHULZ, W. \& SCHWARTZ, H.J. 1995. Appraisal of a project proposal for the Nyabushozi integrated pastoral development project (NIPDEP). Report vol. 2. Project No. 92.2034.4-01.100 Promotion of Veterinary Services, Uganda, GTZ Eschborn (Germany).

DEEM, S.L., PERRY, B.D., KATENDE, J.M., MCDERMOTT, J.J., MAHAN, S.M., MALOO, S.H, MORZARIA, S.P., MUSOKE, A.J. \& ROWLANDS, G.J. 1993. Variations in prevalence of tick-borne diseases in Zebu cattle by agroecological zone: implications for East Coast fever immunization. Preventive Veterinary Medicine, 16:171-187.

FAO 1986. Malignant theileriosis in Southern Province of Zambia-a background paper. 1986. Veterinary Annual Report. Department of Veterinary and Tsetse Control Service. Epidemiology unit, Zambia. 
HEUER, C. 1995. Study design: tick borne diseases in the Ankole Ranching Scheme. Report to the Uganda-German Bilateral Project for the Promotion of Veterinary Services, GTZ Eschborn (Germany).

KATENDE, J.M., TOYE, P., SKILTON, R.A., NENE, V., MORZARIA, S.P. \& MUSOKE, A.J. 1997. An ELISA for detection of Theileria parva antibodies in cattle using a recombinant polymorphic immunodominant molecule. Parasitology Research, 84:408-416.

KRAMER, M.S. 1988. Clinical epidemiology and biostatistics-A primer for clinical investigators and decision-makers. Berlin: Springer Verlag.

MARTIN, S.W., MEEK, A.H. \& WILLEBERG, P. 1987. Veterinary epidemiology. Principles and method. Ames: lowa State University Press.

MCDERMOTT, J.J. SCHUKKEN, Y.H. \& SHOUKRI, M.M. 1994 Study design and analytical methods for data collected from clusters of animals. Preventive Veterinary Medicine, 18:175192.

MOLL, G., LOHDING, A. \& YOUNG, A.S. 1984. Epidemiology of theileriosis in the Trans-Mara division, Kenya: husbandry and disease background and preliminary observation on theileriosis in calves. Preventive Veterinary Medicine, 2:801-831.

MOLL, G., LOHDING, A., YOUNG, A.S. \& LEITCH, B.L. 1986. Epidemiology of theileriosis in calves in an endemic area of Kenya. Veterinary Parasitology, 19:255-273.
MUKHEBI, A.W., PERRY, B.D. \& KRUSKA, R. 1992. Estimated economics of theileriosis control in Africa. Preventive Veterinary Medicine, 12:73-85

NORVAL, R.A.I., PERRY, B.D. \& YOUNG, A.S. 1992. The epidemiology of theileriosis in Africa. London: Academic Press.

PERRY, B.D. \& YOUNG, A.S. 1995. The past and future roles of epidemiology and economics in the control of tick-borne diseases of livestock in Africa: the case of theileriosis. Preventive Veterinary Medicine, 25:107-120.

PERRY, B.D. 1994. Modeling vector-borne disease. Epidemiology and the impact of control programs, in Modeling vectorborne and other parasitic diseases, edited by B.D. Perry \& J.W. Hansen. Proceedings of a Workshop organized by ILRAD in Collaboration with FAO, ILRAD, Nairobi, Kenya, 23-27 November 1992. Nairobi: ILRI.

ROTHMAN, K.J. \& GREENLAND, S. 1998. Modern epidemiolo$g y, 2^{\text {nd }}$ ed. Philadelphia: Lippincott, Raven.

THRUSFIELD, M. 1995. Veterinary epidemiology, $2^{\text {nd }}$ ed. London: Blackwell Science.

WRIGHT, P.F., NILSSON, F., VAN ROOIJ, E.M.A., LELENTA, M. \& JEGGO, M.H. 1993. Standardization and validation of enzyme-linked immunosorbent assay techniques for the detection of antibody in infectious disease diagnosis. Review of Science Techniques, 12:435-450. 